\title{
Operating to remove recurrent colorectal cancer: have we got it right?
}

A randomised controlled trial that remained unpublished for 20 years casts doubt on the survival benefit of further surgery after curative resection of colorectal cancer. Tom Treasure and colleagues tell the story of the first trial restored under the restoring invisible and abandoned trials initiative and discuss what it means today

\section{Tom Treasure professor ${ }^{1}$, Kathryn Monson researcher ${ }^{2}$, Francesca Fiorentino research fellow ${ }^{3}$, Christopher Russell surgeon ${ }^{4}$}

\begin{abstract}
${ }^{1}$ University College London Clinical Operational Research Unit, London, UK; ${ }^{2}$ Sussex Health Outcomes Research and Education in Cancer (SHORE-C), Brighton and Sussex Medical School, University of Sussex, Falmer, UK; ${ }^{3}$ Imperial College, Cardiothoracic Surgery Department, London, UK; ${ }^{4}$ London, UK
\end{abstract}

Old, unpublished clinical trials ordinarily remain unpublished, their results unable to add to the scientific knowledge base, their implications unable to affect practice. But the new restoring invisible and abandoned trials (RIAT) initiative offers a way forward. The RIAT concept allows third parties to publish previously unpublished trials when the original trialists or sponsors fail to do so. ${ }^{1}$ Last June, the editors of $B M J$ and $P L O S$ Medicine called on "researchers and editors to help restore invisible and abandoned trials" by taking unpublished study results and submitting them for publication. ${ }^{2}$ We were among the first to register our intent to RIAT $^{3}$ and have now published a two decade old trial that examined the use of carcinoembryonic antigen (CEA) to prompt "second look" surgery in colorectal cancer. $^{4}$

\section{Role of CEA and second look surgery}

In modern management, a newly diagnosed colorectal cancer is staged, graded, and discussed by a team of surgeons, oncologists, and radiologists with a view to curative surgery if possible. About $16 \%$ of patients operated on will have recurrence of cancer within five years. ${ }^{5}$ Monitoring with the tumour marker CEA is recommended to identify these people as early as possible. The UK's National Institute of Health and Care Excellence recommends CEA tests at least every six months in the first three years plus a minimum of two computed tomograms of the chest, abdomen, and pelvis. ${ }^{6}$ If CEA level is raised and metastases are detected in the liver or lungs, patients are assessed for surgery to remove the metastases with intent to cure.

Before tumour markers were available, there was a drive to monitor for recurrence by "second look" surgery. Second look surgery originated in the 1950s and was promoted in the 1970s.
Asymptomatic patients at high risk of recurrence, such as those who had had affected lymph nodes, were reoperated on at six monthly intervals, with recurrences resected when found. If cancer was found patients were scheduled for further operations (up to a total of six) until the abdomen remained free of cancer. ${ }^{7}$

This approach to management was not without its critics. Colorectal surgeon William Slack wrote in 1982 that this blanket policy might produce some cures but entailed high rates of negative laparotomy and an unacceptable operative mortality rate. ${ }^{8}$ Nevertheless, an analysis of 15 years' experience of

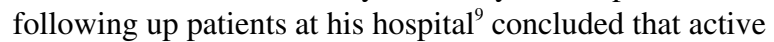
cancer had to be detected earlier than was possible clinically if repeat surgery was to benefit patients. This formed the basis for the CEA second look trial for colorectal cancer, which Slack headed.

By the late 1970s it had been established that raised CEA levels detected cancer recurrence on average four months before it was clinically evident ${ }^{10}$ and had a low false positive rate. ${ }^{11}{ }^{12}$ Testing could spare patients without raised CEA levels needless second look surgery, and early detection was expected to lead to a better prospect of resecting all recurrent disease than when clinical criteria were used to prompt reoperation. It was not clear, however, whether this would result in better survival, which prompted an National Institutes for Health consensus statement calling for trials into CEA with second look surgery in $1981 .{ }^{13}$ Slack and colleagues incorporated CEA testing into a study of second look surgery. The design, by identifying patients with active cancer at the earliest possible point, gave surgery the best chance of being effective. 


\section{What the trial did}

The Carcinoembryonic Antigen Second Look Trial set out to enrol 2000 participants who had had curative resection of colorectal cancer, 500 of whom were expected to have a rise in CEA level indicative of recurrence on regular monitoring. Half of those with a CEA rise were randomised to immediate second look surgery and half to continued routine clinical care, which at the time was clinical review every three months for the first year, then six monthly until there was clinical suspicion of recurrence. At this point second look surgery was also to be considered. The hypothesis was that CEA monitoring would increase the success rate of second look procedures, in terms of macroscopic clearance, from $25 \%$ to $55 \%$. The trial was powered to detect a resulting improvement in survival from $10 \%$ to $22 \%$ at five years. ${ }^{84}$ According to the protocol surgeons were to resect any recurrence at the surgical site, in the peritoneum, or in lymph nodes. They were also to mobilise the liver to identify and resect metastases.

The trial started in 1982 and by 1993 had recruited 1447 of the intended 2000 participants and randomised 216 when it was stopped early. The data monitoring committee thought that it was highly unlikely that any survival advantage would ever be shown. ${ }^{14}{ }^{15}$ When the trial was unblinded it was found that there were more deaths in the active arm than the control arm (91 v 88 ; relative risk $=1.16,95 \%$ confidence interval 0.87 to 1.37$){ }^{4}$ This important finding might have influenced practice, but it remained unpublished for 20 years.

\section{How the trial was lost}

The study ran for longer than expected and was affected by various organisational changes. Recruitment was slow, and only $15 \%$ of patients met the stringent criteria for randomisation rather than the $25 \%$ predicted. During the course of recruitment, the trial unit moved from King's College Hospital, London, to University College London. Slack, the chief investigator, retired and John Northover, who had been a driving force throughout, took on that role.

Although the study was stopped early, there was a clear intention to publish. The primary outcome, survival, was recorded in an abandoned version of the manuscript dated 6 July 1994 that we found in the archive and had been revealed publicly in a letter to JAMA ${ }^{14}$ and in a conference abstract in the British Journal of Cancer. ${ }^{15}$ The methods and results appear complete in the manuscript prepared for submission in 1994 but the discussion halts after three lines with a comment about the identification and analysis of subsets. Our interpretation is that differences over attempts to perform post hoc exploratory analyses may have led to the breakdown of the efforts to publish in 1994.

The data then languished until after the sudden death of the trial statistician, Kenneth MacRae, in April 2002. The study was referred to two trial statisticians outside the trial centre, who looked at the electronic files around 2003. They failed to access the data to their satisfaction, deeming them "corrupted," and a decision was made to not reopen the analysis.

\section{Piecing the trial back together}

In the face of increasing referrals for pulmonary metastasectomy in colorectal trials a proposal was made for a trial in $2009^{16}$ comparing active monitoring with active monitoring plus pulmonary metastasectomy in patients who had curative resection for colorectal cancer, the Pulmonary Metastasectomy in Colorectal Cancer (PulMiCC) trial. ${ }^{17}$ The trial was designed to resolve the paradox that patients who had pulmonary metastases detected by CEA were being referred for metastasectomy despite consistent reports that raised CEA levels were associated with poor survival after pulmonary metastasectomy. ${ }^{18}{ }^{19}$ As the data from the second look study were relevant to PulMiCC, in August 2009 we asked Northover what had happened to the results. He believed the data to be irretrievably lost. Michael Baum, who headed the trial's data monitoring committee, thought the same. In October 2009 we contacted others involved at the time. The UCL clinical trials centre response was that the data were corrupted.

One of us, KM, had recently left UCL and was aware of the trial's history and electronic database problems but she knew that the files were still in the unit (fig $1 \Downarrow$ ). On 6 November 2009 TT asked the current director of the trial centre, Jonathan Ledermann, for access to the data. We were eventually given access in March 2011. Staff at the trial centre retrieved the archived electronic files and the centre's director authorised our access to an anonymised copy, saying, "The problem is that the data are in a total mess."

We discovered that the data were not corrupted but difficult to analyse. The 1980s had been a time of rapid development in computing and software, demanding steep learning curves in its implementation. File transfers by temporary staff and various informal codes inserted into the data had made data extraction seem impossible at first sight, but we were able to restore trustworthy and usable records for 1446 of the 1447 participants. We then updated the survival data to September 2011. The data available in February 1993 were that 91/108 patients had died in the "aggressive" arm and 88/108 in the "conventional" arm (relative risk $=1.16,95 \%$ confidence interval 0.87 to 1.37 ). Our updated analysis confirms that there is no hint of a survival advantage associated with knowledge of the CEA (fig $2 \Downarrow$ ). ${ }^{4}$

We spoke to as many as we could find of those listed in the trial documents at the outset ${ }^{8}$ and in the draft manuscript to check we had made sense of the data and had not overlooked anything. It was clear that the trial team had broken up in disarray, but those we spoke to regretted that the trial had not been published and supported us now doing so.

\section{What the trial means today}

By the time that the CEA trial closed clinical practice had shifted. CEA testing had become commonplace after curative resection of colorectal cancer, and liver and lung metastasectomy had been adopted based on observational evidence (box). ${ }^{20}{ }^{21}$ In 1992 the Mayo Clinic proposed that a randomised trial should be done to determine the survival difference resulting from resection of liver metastases compared with no resection. ${ }^{22}$ It proposed that if the difference in five year survival with liver metastasectomy was $25 \%$ versus $1 \%$, as was being claimed, only 36 randomised patients would be needed to confirm an effect. However, the trial did not take place because of a general view that "it would be difficult to obtain informed consent from patients randomised to no treatment despite resectable disease and, in view of reported results of surgical resection, it is unlikely that ethical committees would agree to such a trial., ${ }^{35}$

Although the CEA second look trial found no benefit from an overall policy of detecting and resecting disseminated cancer, organ specific resection of metastases has become common practice. The recent Follow-up After Colorectal Surgery (FACS) trial $^{5}$ tested the effectiveness of intensive monitoring with CEA testing, computed tomography, or both compared with no scheduled follow-up except a single computed tomogram of the chest, abdomen, and pelvis at 12 to 18 months if requested at study entry by the hospital clinician. ${ }^{5}$ There was a higher death 


\section{How the CEA Second Look Trial was left behind by events}

1954: Wangensteen advocated second look surgery in asymptomatic patients after colorectal cancer ${ }^{7}$

1971-78: Resection of recurrent cancer after potentially curative resection of colorectal cancer was believed to sometimes lead to "cure"23-25

1974-80: CEA was shown to detect asymptomatic recurrence of colorectal cancer ${ }^{1026-29}$

1981: National Institutes for Health consensus called for a trial of $C E A^{30}$

1982: Slack and Northover started the CEA Second Look trial ${ }^{8}$

1982-89: Hughes published an international registry of hepatic metastases resection from 24 institutions in North America, England, and Germany. After excluding 30 day mortality, they reported five year survival of $30 \%{ }^{31-34}$

1990-91: Surgeons at Erlangen University Hospital, Germany, publish their results for liver resections. After exclusion of $5.5 \%$ postoperative death five year survival was $39 \%{ }^{35-37}$

1992: Memorial Sloan-Kettering Cancer Center in New York published 10 year results of pulmonary metastasectomy in colorectal cancer. Five and 10 year survival rates were $40 \%$ and $30 \%$, respectively ${ }^{38}$

1992: Mayo Clinic surgeons publish the power calculation for a randomised trial of liver resection suggesting that 36 patients would have been sufficient to prove benefit ${ }^{39}$

1994: CEA Second Look Trial results available ${ }^{14} 15$

1994: Erlangen group wrote in the Lancet, "The benefit in outcome provided by resection of colorectal liver metastases had been clearly demonstrated' based on $30 \%$ five-year disease-free survival amongst the $10-20 \%$ of their patients selected for this surgery"

1994: Erlangen group wrote "trials on ... effectiveness of hepatic resection for metastatic colorectal cancer [would be] not only obsolete but unethical"41

rate in the intensive monitoring group $(18.2 \%(164 / 901) v 15.9 \%$ (48/301); difference $2.3 \%, 95 \%$ CI $-2.6 \%$ to $7.1 \%$ ). The study had originally intended to estimate the difference in overall survival but "in 2007 when it became clear that we could not recruit the number of participants necessary to estimate an effect on overall survival with adequate statistical power" the primary outcome was changed to "surgical treatment with curative intent." This was not, in fact, "curative" surgery since it is not associated with higher survival rates. The FACS trial results are in accordance with the absence of survival benefit found in the CEA second look trial in 1994.

Methods of detection, imaging, and surgical resection have changed over the intervening 20 years and those committed to resecting metastases may well regard the results of the restored CEA Second Look trial as irrelevant. We take a different view. We do not believe that the findings of the CEA trial can be readily discounted but rather consider that the onus is on those promoting unproved operations to test their effectiveness in controlled trials.

Contrary to a commonly held view among surgeons, patients are not the obstacle to trials. In the National Cancer Patient Experience Survey of over 70000 patients in the British National Health Service around two thirds did not have cancer research discussed with them, though half would have preferred that it had been..$^{42}$ Of the patients who were made aware of research, two thirds went on to be included in research studies. Patients are entitled to a voice, but are we listening?

We believe that the new evidence should fuel uncertainty about present day second look surgery for colorectal cancer in its various forms and hope that it will give some encouragement to undertake the randomised trials that are needed. Within the NHS these studies could be promoted by the National Institute for Health Research and the National Cancer Research Institute.

We thank Jonathan Ledermann, director of the centre where the CEA files were stored, and Sharon Forsyth for her help in accessing the CEA trial data and updating the Office for National Statistics records for death registration. We authors also met the following people who were members of the 1982 working party for the study or listed as contributors in the 1994 draft manuscript: M Baum, R H J Begent, $\mathrm{H}$ Ellis, J Houghton, M Irving, C A Lennon, J M A Northover, W W Slack, and C $B$ Wood. We thank them for frank discussions concerning the progress of the study and the factors leading to its closure and the abandonment of publication.
Contributors and sources: TT is chief investigator and CR is chair of the trial steering committee of the Pulmonary Metastasectomy in Colorectal Cancer (PuIMiCC) trial. They both worked at the Middlesex Hospital and knew about the CEA Second Look Trial from its inception in 1982. KM works on the PuIMiCC trial and was previously in the UCL Trials Unit during enrolment to the CEA Second-Look Trial, but she had not worked on it. KM and TT restored the data under the leadership of CR. FF worked with TT on a research programme of systematic reviews and analytical studies related to surgery. She did the analysis. The authors interviewed as many of those involved with the second look trial as could be found.

Competing interests: We have read and understood the BMJ Group policy on declaration of interests and have no relevant interests to declare.

Provenance and peer review: Not commissioned; externally peer reviewed.

1 Doshi P, Dickersin K, Healy D, Vedula SS, Jefferson T. Restoring invisible and abandoned trials: a call for people to publish the findings. BMJ 2013;346:f2865.

2 Loder E, Godlee F, Barbour V, Winker M: Restoring the integrity of the clinical trial evidence base. BMJ 2013;346:\{3601.

3 Treasure T, Russell RCG. Electronic response to: Doshi et al, Restoring invisible and abandoned trials: a call for people to publish the findings. BMJ 2013. www.bmj.com/ content/346/bmj.f2865/rr/668952.

4 Treasure T, Monson K, Fiorentino F, Russell RCG. The CEA Second Look Trial: a randomised controlled trial of carcinoembryonic antigen prompted reoperation for recurrent colorectal cancer. BMJ Open 2014;4:e004385.

5 Primrose JN, Perera R, Gray A, Rose P, Fuller A, Corkhill A, et al. Effect of 3 to 5 years of scheduled CEA and CT follow-up to detect recurrence of colorectal cancer: the FACS randomized clinical trial. JAMA 2014;311:263-70.

6 Poston G, Guideline Development Group. Colorectal cancer: the diagnosis and management of colorectal cancer. National Institute for Health and Clinical Excellence, 2011. http://guidance.nice.org.uk/CG131.

7 Wangensteen O, Lewis F, Arhelger S, Muller J, Maclean L. An interim report upon the second look procedure for cancer of the stomach, colon, and rectum and for limited intraperitoneal carcinosis. Surg Gynecol Obstet 1954;99:257-67.

8 Slack W, Bagshawe K, Baum M, Ellis H, Northover J, Wood C, et al. Protocol: a multicentre trial to evaluate the use of serial carcinoembryonic antigen assay as the prime indicator for second-look surgery in recurrent colorectal cancer. (Trial Archive); 1982. doi:10.5061 dryad.k8t8b.

9 Cochrane JP, Williams JT, Faber RG, Slack WW. Value of outpatient follow-up after curative surgery for carcinoma of the large bowel. BMJ 1980;280:593-5.

10 Staab HJ, Anderer FA, Stumpf E, Fischer R. Carcinoembryonic antigen follow-up and selection of patients for second-look operation in management of gastrointestinal carcinoma. J Surg Oncol 1978;10:273-82.

11 Martin EW Jr, Cooperman M, King G, Rinker L, Carey LC, Minton JP. A retrospective and prospective study of serial CEA determinations in the early detection of recurrent colon cancer. Am J Surg 1979;137:167-9.

12 Meeker WR Jr. The use and abuse of CEA test in clinical practice. Cancer 1978;41:854-62.

13 Goldenberg DM. Carcinoembryonic antigen: its role as a marker in the management of cancer. Summary of an NIH consensus statement. BMJ 1981;282:373-5.

14 Northover J, Houghton J, Lennon T. CEA to detect recurrence of colon cancer [Letter] JAMA 1994;272:31

15 Lennon T, Houghton J, Northover J. Post operative CEA monitoring and second-look surgery in colorectal cancer: trial results [abstract]. Br J Cancer 1994;70(suppl 22):16.

16 Treasure T, Fallowfield L, Farewell V, Ferry D, Lees B, Leonard P, et al. Pulmonary metastasectomy in colorectal cancer: time for a trial. Eur J Surg Oncol 2009;35:686-9. 
17 Treasure T, Fallowfield L, Lees B. Pulmonary metastasectomy in colorectal cancer: the PulMiCC trial. J Thorac Oncol 2010;5:S203-6.

18 Treasure T. Carcinoembryonic antigen: its place in decision making for pulmonary metastasectomy in colorectal cancer. J Thorac Oncol 2010;5:S179-81.

19 Fiorentino F, Hunt I, Teoh K, Treasure T, Utley M. Pulmonary metastasectomy in colorecta cancer: a systematic review and quantitative synthesis. J R Soc Med 2010;103:60-6.

20 Grunhagen D, Jones RP, Treasure T, Vasilakis C, Poston GJ. The history of adoption of hepatic resection for metastatic colorectal cancer: 1984-95. Crit Rev Oncol Hematol 2013;86:222-31.

21 Fiorentino $F$, Vasilakis $C$, Treasure T. Clinical reports of pulmonary metastasectomy for colorectal cancer: a citation network analysis. Br J Cancer 2011;104:1085-97.

22 Rosen CB, Nagorney DM, Taswell HF, Helgeson SL, Ilstrup DM, Van Heerden JA, et al. Perioperative blood transfusion and determinants of survival after liver resection for metastatic colorectal carcinoma. Ann Surg 1992;216:493-504.

23 Polk HC Jr, Spratt JS Jr. Recurrent colorectal carcinoma: detection, treatment, and other considerations. Surgery 1971;69:9-23.

24 Ellis $\mathrm{H}$. Is a second look operation justified in suspected recurrences after abdominal cancer surgery? Br J Surg 1975;62:830-2.

25 Welch JP, Donaldson GA. Detection and treatment of recurrent cancer of the colon and rectum. Am J Surg 1978;135:505-11.

26 Mach JP, Jaeger P, Bertholet MM, Ruegsegger CH, Loosli RM, Pettavel J. Detection of recurrence of large-bowel carcinoma by radioimmunoassay of circulating carcinoembryonic antigen (CEA). Lancet 1974;304:535-540.

27 Mackay AM, Patel S, Carter S, Stevens U, Laurence DJ, Cooper EH, et al. Role of serial plasma CEA assays in detection of recurrent and metastatic colorectal carcinomas. BMJ 1974;4:382-5.

28 Nicholson JR, Aust JC. Rising carcinoembryonic antigen titers in colorectal carcinoma: an indication for the second-look procedure. Dis Colon Rectum 1978;21:163-4.

29 Martin EW, Cooperman M, Carey LC, Minton JP. Sixty second-look procedures indicated primarily by rise in serial carcinoembryonic antigen. J Surg Res 1980;28:389-94.

30 Goldenberg DM. Carcinoembryonic antigen: its role as a marker in the management of cancer. Summary of an NIH consensus statement. BMJ 1981;282:373-5.
31 Hughes ES, McConchie IH, McDermott FT, Johnson WR, Price AB. Resection of lung metastases in large bowel cancer. Br J Surg 1982;69:410-2

32 Hughes KS, Simon R, Songhorabodi S, Adson MA, Ilstrup DM, Fortner JG, et al. Resection of the liver for colorectal carcinoma metastases: a multi-institutional study of patterns of recurrence. Surgery 1986;100:278-84.

33 Hughes K, Registry of hepatic metastases: resection of the liver for colorectal carcinoma metastases: a multi-institutional study of indications for resection. Surgery 1988:103:278-88

34 Hughes K, Scheele J, Sugarbaker PH. Surgery for colorectal cancer metastatic to the liver. Optimizing the results of treatment. Surg Clin North Am 1989;69:339-59.

35 Scheele J, Stangl R, Altendorf-Hofmann A. Hepatic metastases from colorectal carcinoma: impact of surgical resection on the natural history. Br J Surg 1990;77:1241-6.

36 Scheele J, Altendorf-Hofmann A, Stangl R, Gall FP. Pulmonary resection for metastatic colon and upper rectum cancer. Is it useful? Dis Colon Rectum 1990:33:745-52.

37 Scheele J, Stangl R, Altendorf-Hofmann A, Gall FP. Indicators of prognosis after hepatic resection for colorectal secondaries. Surgery 1991;110:13-29.

38 McCormack PM, Burt ME, Bains MS, Martini N, Rusch VW, Ginsberg RJ. Lung resection for colorectal metastases. 10-year results. Arch Surg 1992;127:1403-6.

39 Rosen CB, Nagorney DM, Taswell HF, Helgeson SL, Ilstrup DM, Van Heerden JA, et al. Perioperative blood transfusion and determinants of survival after liver resection for metastatic colorectal carcinoma. Ann Surg 1992;216:493-504.

40 Stangl R, Altendorf-Hofmann A, Charnley RM, Scheele J. Factors influencing the natural history of colorectal liver metastases. Lancet 1994;343:1405-10.

41 Scheele J, Stangl R, Altendorf-Hofmann A, Paul M. Resection of colorectal liver metastases. World J Surg 1995;19:59-71.

42 NHS. National cancer patient experience survey. 2012. www.gov.uk/government/uploads/ system/uploads/attachment_data/file/212860/Cancer-Patient-Experience-Survey-NationalReport-2011-12.pdf.

Cite this as: BMJ 2014;348:g2085

(c) BMJ Publishing Group Ltd 2014 


\section{Figures}

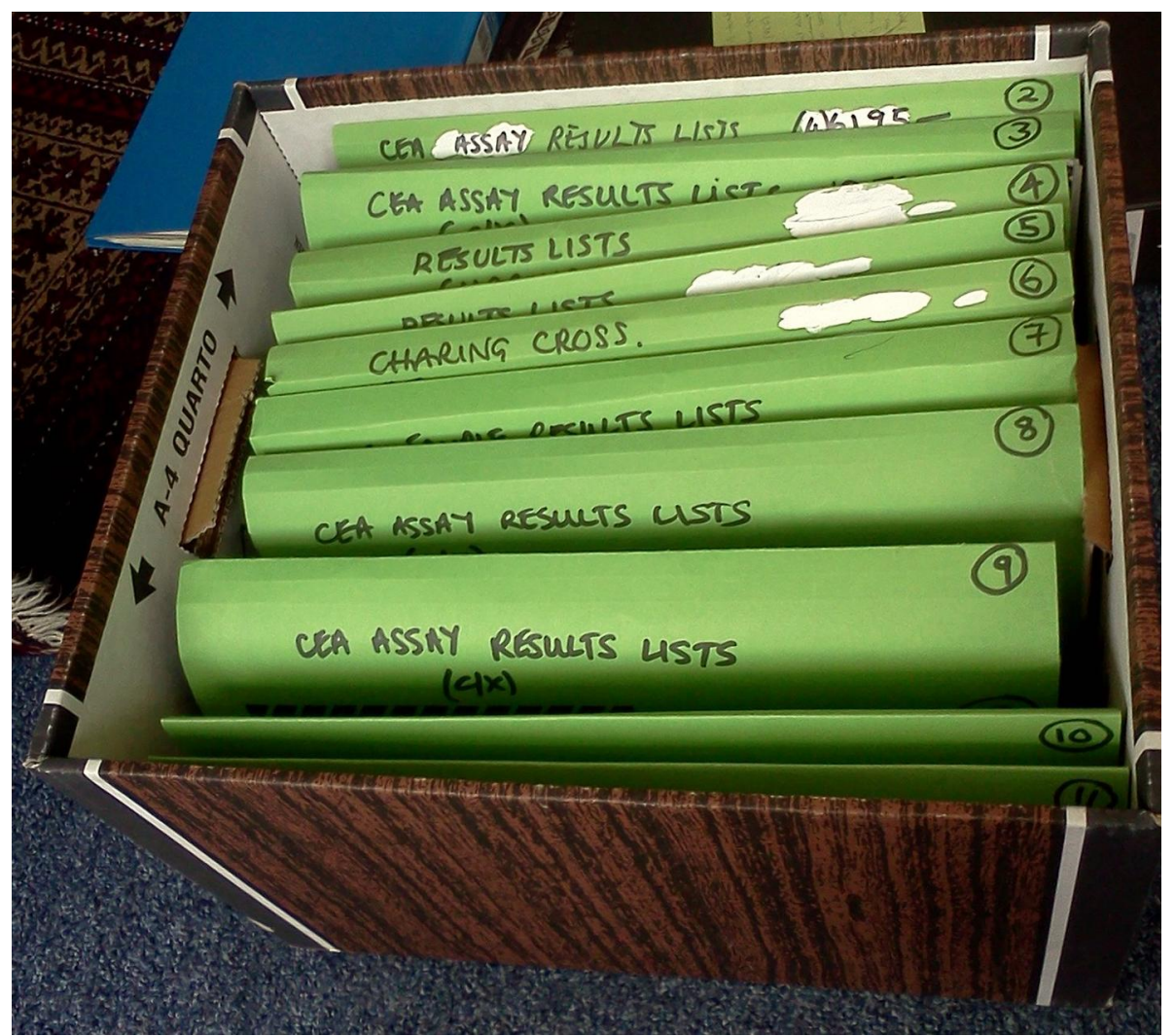

Fig 1 Rediscovered data files for the CEA second look study

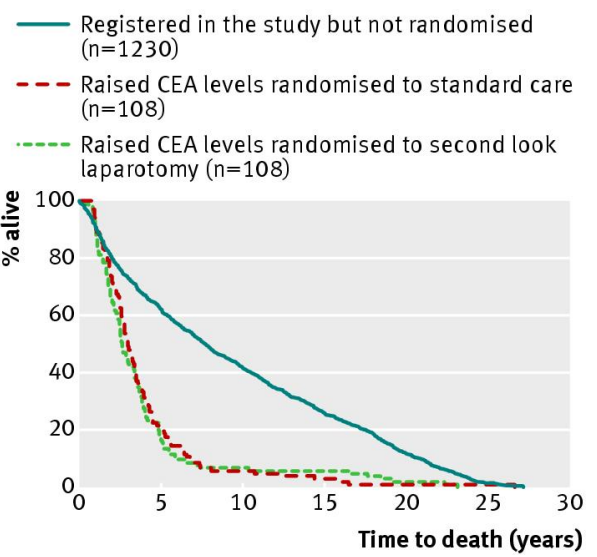

Fig 2 Kaplan-Meier analysis of survival among participants in the Carcinoembryonic Antigen (CEA) Second Look Trial ${ }^{4}$ 